\title{
Neutrophil Gelatinase-Associated Lipocalin and Matrix Metalloproteinase-9 as Potential Biomarkers for Stroke: A Pilot Study
}

Yi-Chinn Weng and Wen-Hai Chou*

Department of Biological Sciences and School of Biomedical Sciences, Kent State University, Kent, Ohio, USA

*Corresponding author: Wen-Hai Chou, Department of Biological Sciences, Kent State University, Kent, Ohio 44242, USA; Tel.: 330-672-2979, Fax: 330-672-3713, Email: wchou2@kent.edu

Received date: March 13, 2015; Accepted date: March 30, 2015; Published date: April 04, 2015

Copyright: $\odot 2015$ Chou WH. This is an open-access article distributed under the terms of the Creative Commons Attribution License, which permits unrestricted use, distribution, and reproduction in any medium, provided the original author and source are credited.

\begin{abstract}
Recent evidence suggests that neutrophil gelatinase-associated lipocalin (NGAL) is acutely induced after ischemic stroke. Matrix metalloproteinase-9 (MMP-9) has been associated with hemorrhagic transformation possibly by promoting the degradation of extracellular matrix of cerebral blood vessels. NGAL forms heterodimer with MMP- 9 and stabilizes the enzymatic activities of MMP-9 by protecting it from degradation. In the present study, we determined the concentrations of NGAL, MMP-9 and the heterodimeric complex in the sera of patients with ischemic and hemorrhagic stroke as well as healthy subjects by Western blotting, affinity purification, and ELISA. Our results show that the serum levels of NGAL, MMP-9, and NGAL-MMP-9 complex in hemorrhagic stroke patients were significantly higher than the ischemic stroke and control subjects. The data suggest a potential usage of NGAL, MMP-9 and the heterodimeric complex as biomarkers to detect hemorrhagic stroke and distinguish it from ischemic stroke.
\end{abstract}

Keywords: NGAL; Lipocalin-2; MMP-9; Biomarker; Stroke

\section{Introduction}

Stroke is the most common neurological disease worldwide [1,2]. About $75 \%$ of those who survive will suffer long-term impairment of movement, sensation, memory, or cognition. Ischemic stroke represents $80 \%$ of all cases, and results from interruption of blood supply to a portion of the brain. Current acute treatment aims to restore the blood supply by administering fibrinolytic agents such as tissue plasminogen activator (tPA) [3]. Less than $5 \%$ of stroke victims receive these drugs because they must be given within the first 4.5 hours of stroke onset. If given later, such treatment does not appear to be beneficial, and can increase the risk of serious side effects such as hemorrhage [4]. Hemorrhagic transformation is a serious complication following thrombolytic therapy, producing severe neurological deficits and a high rate of mortality. The clinical problem at hand is how to make the diagnosis early, extend the time window for tPA, reduce the risk of cerebral hemorrhage, and ultimately increase the overall efficacy of thrombolytic therapy $[5,6]$.

NGAL, also known as lipocalin-2 (LCN2) or 24p3, is a member of lipocalin protein family $[7,8]$. Elevated plasma levels of NGAL have been detected in patients suffering from acute ischemic stroke [9-11]. We recently found that NGAL protein is induced acutely after ischemic stroke in mice and is an important mediator of reperfusion injury [12]. Increased NGAL levels were observed in mouse serum as early as one hour after stroke, continued to rise between 4 and 23 hours, and were diminished by 48 to 72 hours. The early induction of NGAL suggests the possibility of using NGAL as a biomarker for stroke.

NGAL was originally identified as a $25 \mathrm{kDa}$ protein covalently associated with MMP-9 $(92 \mathrm{kDa})$ [7,13]. Formation of this heterodimer enhances and stabilizes the gelatinolytic activity of MMP-9 by preventing MMP-9 from degradation [14-16]. MMP-9 is a member of the matrix metalloproteinase family that proteolytically degrades the extracellular matrix of cerebral blood vessels [17]. Aberrant upregulation of MMP-9 has been implicated in the deterioration of the blood-brain barrier (BBB), worsening of cerebral infarction and functional outcome after tPA treatments [18-20]. Moreover, tPA directly stimulates the release and activation of MMP-9 [21-23]. Based on these studies, we decided to investigate whether NGAL, MMP-9 and NGAL-MMP-9 complex can be used as biomarkers to identify ischemic and hemorrhagic strokes.

\section{Materials and Methods}

\section{Patient sera}

The serum samples of patients with hemorrhagic stroke, ischemic stroke, and healthy aged-matched subjects were purchased from ProteoGenex (Culver City, CA, USA). Fifteen samples were obtained for each group. The sera were collected within 24 hours after the diagnosis. Prior to participation, written informed consent was obtained from all subjects. The study was performed in accordance with the Declaration of Helsinki. Patient information (e.g. age and sex) was obtained from ProteoGenex. Healthy subjects with hypertension history were excluded from studies.

\section{Enzyme-linked immunosorbent assay (ELISA)}

The levels of NGAL, MMP-9 and NGAL-MMP-9 complex in the sera were quantified following the manufacturer's protocol for human lipocalin-2/NGAL Quantikine ELISA kit, human MMP-9 Quantikine ELISA kit, and human MMP-9/NGAL Complex Quantikine ELISA Kit (R\&D, Minneapolis, MN, USA). 
Citation: Weng YC, Chou WH (2015) Neutrophil Gelatinase-Associated Lipocalin and Matrix Metalloproteinase-9 as Potential Biomarkers for Stroke: A Pilot Study. J Neurol Neurophysiol 6: 278. doi:10.4172/2155-9562.1000278

Page 2 of 5

\section{Gelatin affinity purification of MMP-9 and NGAL-MMP-9 complex}

Gelatin sepharose 4B (GE Healthcare, Piscataway, NJ, USA) was washed three times with cold TNC buffer $(50 \mathrm{mM}$ Tris- $\mathrm{HCl} \mathrm{pH} 7.5$, $150 \mathrm{mM} \mathrm{NaCl}, 10 \mathrm{mM} \mathrm{CaCl} 2,0.02 \% \mathrm{NaN} 3)$. The protein concentrations of human sera were measured by Bradford assays and the sera were diluted in the TNC buffer containing Halt Protease and Phosphatase Inhibitor and Pepstatin A Protease Inhibitor (Pierce, Rockford, IL, USA) to a final concentration of $4 \mathrm{ug} / \mathrm{ul}$. The diluted human sera were incubated with the washed gelatin sepharose $4 \mathrm{~B}$ for 2 hours with rotation at $4^{\circ} \mathrm{C}$. The mixture was centrifuged at $5000 \mathrm{rpm}$ for 5 minutes at $4^{\circ} \mathrm{C}$ and the supernatant was removed. The gelatin sepharose $4 \mathrm{~B}$ was washed with the TNC buffer three times to remove unbound proteins. The gelatin sepharose 4B with bound MMP-9 and the associated proteins were resuspended in SDS sample buffer and analyzed by Western blotting.

\section{Western blot}

Protein samples were separated by NuPAGE 4-12\% Bis-Tris gels (Invitrogen, Carlsbad, CA, USA), transferred to PVDF membranes, and analyzed by Western blotting using goat anti-NGAL (1:1000, R\&D, Minneapolis, MN, USA) and rabbit anti-MMP-9 (1:1000, Abcam, Cambridge, MA, USA) antibodies. Immunoreactive bands were detected by enhanced chemiluminescence (ECL) (Pierce, Rockford, IL, USA) and imaged using a Luminescent Image Analyzer LAS-3000 (Fujifilm, Tokyo, Japan).

\section{Statistical analysis}

Quantitative data were expressed as mean \pm SEM and analyzed using Prism 5.0 (GraphPad Software, La Jolla, CA, USA). One-way ANOVA with Newman-Keuls post hoc tests were employed to determine statistical significance between means. The values of $P$ less than 0.05 were considered to be statistically significant.

\section{Results}

\section{Determine the level of NGAL in human sera}

We and others found that the level of NGAL is elevated after ischemic stroke in mouse sera [12] and human plasma [9-11]. To assess the level of NGAL in different types of strokes, we analyzed patient sera of ischemic and hemorrhagic strokes by Western blotting with the antibody against NGAL.

\begin{tabular}{|l|l|l|l|}
\hline Characteristics & Normal donor & $\begin{array}{l}\text { Ischaemic } \\
\text { Stroke }\end{array}$ & $\begin{array}{l}\text { Haemorrhagic } \\
\text { Stroke }\end{array}$ \\
\hline Age range & $67-85$ & $59-84$ & $61-90$ \\
\hline Median age & 75 & 72 & 82 \\
\hline Female & 12 & 12 & 14 \\
\hline Male & 3 & 3 & 1 \\
\hline
\end{tabular}

Table 1: Characteristics of normal donors and stroke patients

The sera of aged-matched healthy donors were used as controls. Table 1 shows the characteristics of enrolled subjects. An immunoreactive band of NGAL $(25 \mathrm{kDa})$ was detected in the human sera by Western blotting (Figure 1A). The intensities of NGAL protein bands were slightly higher in the sera of ischemic stroke patients and considerably stronger in hemorrhagic stroke patients when compared with the controls. To confirm the results of Western blotting, we measured the concentrations of NGAL by ELISA (Figure 1B). The serum level of NGAL was $111.8 \pm 8.6 \mathrm{ng} / \mathrm{ml}$ in normal donor and $152.7 \pm 11.4 \mathrm{ng} / \mathrm{ml}$ in ischemic stroke patients. Interestingly, the NGAL level was increased almost two folds to $213.8 \pm 34.0 \mathrm{ng} / \mathrm{ml}$ in hemorrhagic stroke patients. The serum level of NGAL in hemorrhagic stroke patients was significantly higher than the ischemic stroke patients and healthy donors $(\mathrm{P}<0.05)$.

Figure 1
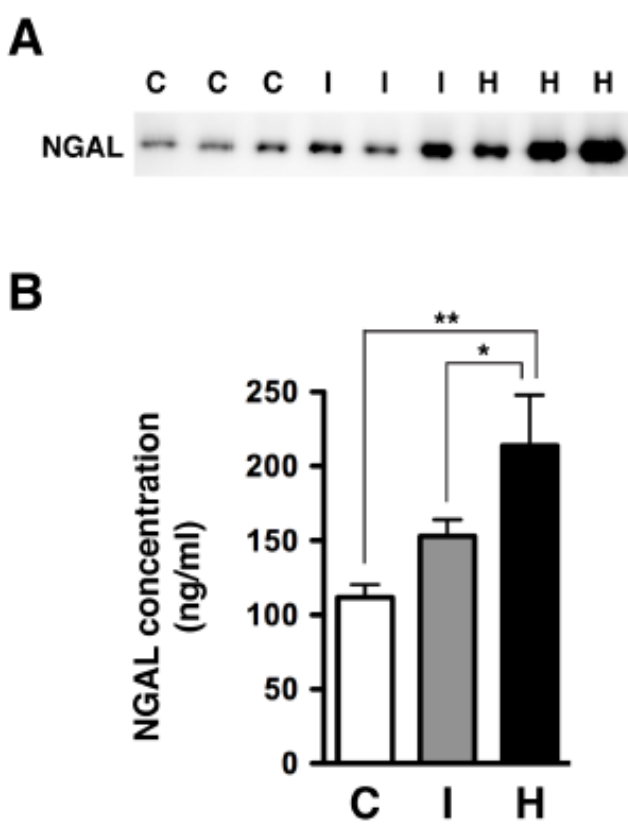

Figure 1: Expression of NGAL in the sera of normal donors and stroke patients. (A) A representative Western blot shows the expression NGAL in the sera of control (C), ischemic (I), and hemorrhagic $(\mathrm{H})$ stroke patients. (B) The levels of NGAL in the sera of normal donors and stroke patients were measured by ELISA. Data were analyzed by a one-way ANOVA with NewmanKeuls post hoc tests. There was a significant induction of NGAL in hemorrhagic stroke patients $\left({ }^{\star} \mathrm{P}<0.05\right)$ compared with normal donors and ischemic stroke patients.

\section{Determine the level of MMP-9 in human sera}

Previous studies have detected elevated serum levels of MMP-9 in patients with ischemic stroke [24] and intracranial hemorrhage [25]. To verify these findings, we performed Western blotting and detected a $92 \mathrm{kDa}$ immunoreactive band of MMP-9 (Figure 2A). The MMP-9 protein bands detected in hemorrhagic stroke patients appeared darker than the ischemic stroke and control sera. The concentrations of MMP-9 determined by ELISA correlated with the Western blotting 
Citation: Weng YC, Chou WH (2015) Neutrophil Gelatinase-Associated Lipocalin and Matrix Metalloproteinase-9 as Potential Biomarkers for Stroke: A Pilot Study. J Neurol Neurophysiol 6: 278. doi:10.4172/2155-9562.1000278

Page 3 of 5

(Figure 2B). The MMP-9 level in the sera of hemorrhagic stroke patients $(1112 \pm 148.6 \mathrm{ng} / \mathrm{ml})$ was significantly higher than the ischemic stroke patients $(715.6 \pm 81.2 \mathrm{ng} / \mathrm{ml})$ and controls $(629.3 \pm 60.8$ $\mathrm{ng} / \mathrm{ml})(\mathrm{P}<0.05)$.

\section{Figure 2}

A

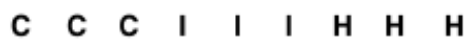

MMP-9

\section{B}

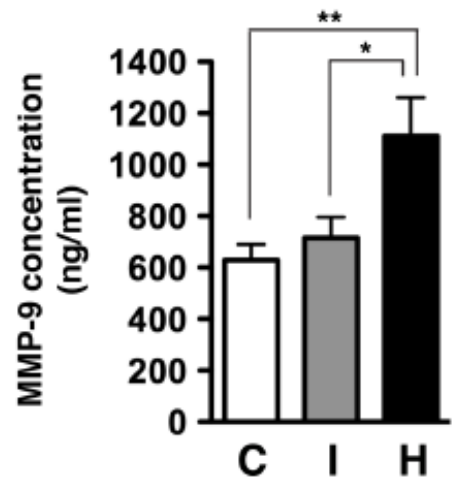

Figure 2: Expression of MMP-9 in the sera of normal donors and stroke patients. (A) A representative Western blot shows the expression of MMP-9 in the sera of control (C), ischemic (I), and hemorrhagic (H) stroke patients. (B) The levels of NGAL in the sera of normal donors and stroke patients were measured by ELISA. Data were analyzed by a oneway ANOVA with NewmanKeuls post hoc tests. There was a significant induction of MMP-9 in hemorrhagic stroke patients $\left({ }^{\star} \mathrm{P}<0.05\right)$ compared with normal donors and Ischemic stroke patients.

\section{Determine the level of NGAL-MMP-9 complex in human sera}

NGAL dimerizes with MMP-9 via an intermolecular disulfide bond and stabilizes the enzymatic activity of MMP-9 [14-16]. To assess the level of NGAL-MMP-9 complex, we isolated MMP-9 and the complex by gelatin affinity purification. The NGAL associated with MMP-9 was separated by SDS-PAGE and determined by Western blotting to represent the level of NGAL-MMP-9 complex. The co-purified NGAL immunoreactive protein bands showed stronger intensity in the sera of hemorrhagic stroke patients than the ischemic stroke and healthy controls. To quantify the level of NGAL-MMP-9 complex, we performed ELISA and found that the level of complex was significantly elevated in the sera of hemorrhagic stroke patients $(109.6 \pm 30.2$ $\mathrm{ng} / \mathrm{ml})$ than in the controls $(52.7 \pm 7.3 \mathrm{ng} / \mathrm{ml})$ and ischemic stroke patients $(45.0 \pm 8.7 \mathrm{ng} / \mathrm{ml})(\mathrm{P}<0.05)$. Interestingly, the level of complex was slightly reduced in the ischemic stroke patients when compared with the controls (Figure 3).
Figure 3

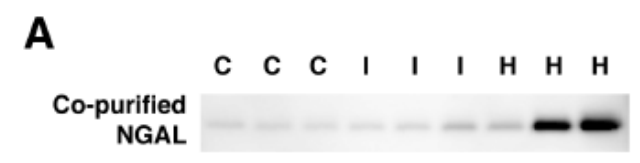

B

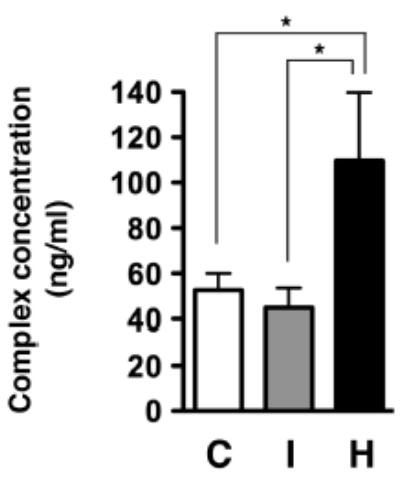

Figure 3: Expression of NGAL-MMP-9 complex in the sera of normal donors and stroke patients. (A) A representative Western blot shows the expression of NGAL-MMP-9 complex in the sera of control (C), ischemic (I), and hemorrhagic (H) stroke patients. (B) The levels of NGAL-MMP-9 complex in the sera of normal donors and stroke patients were measured by ELISA. Data were analyzed by a one-way ANOVA with Newman-Keuls post hoc tests. 13 There was a significant induction of NGAL-MMP-9 complex in hemorrhagic stroke patients $\left({ }^{*} \mathrm{P}<0.05\right)$ compared with normal donors and ischemic stroke patients.

\section{Discussion}

This pilot study demonstrates that the levels of NGAL, MMP-9 and their complex in patients with hemorrhagic stroke were significantly higher than ischemic stroke and healthy controls, suggesting an exciting potential of using these proteins as stroke biomarkers. Since only 15 subjects were included in each group, future research with a larger number of stroke patients is necessary to determine optimum cut-offs for stroke detection and classification.

Nearly $95 \%$ of patients with acute ischemic stroke are excluded from the thrombolytic therapy because they have missed the 4.5-hour time window $[3,4]$. The late thrombolytic treatment may cause intracerebral hemorrhage and death. Neuroimaging techniques such as magnetic resonance imaging (MRI) and computed tomography scan (CT) have proven to be invaluable for stroke diagnosis $[26,27]$. However, many hospitals are not equipped with the specialized radiologic equipment due to the costs of equipment and personnel. These observations suggest that the availability of a rapid assay using blood biomarkers would be highly valuable in identifying appropriate diagnosis, treatment, and prognosis, as well as avoid over utilization of resources and provide cost effective care $[28,29]$. Although there is no 
biomarker for stroke that is routinely used in clinical practice, developing such a test is vital and ongoing [30,31]. To date, a number of proteins (S100B, MMP-9, vWF, BDNF, VCAM) show promise as biomarkers in stroke, however sufficient sensitivity and specificity for use in clinical practice has not been achieved [28,32]. Recently, a panel of multiple biomarkers instead of a single marker has been used to improve the diagnostic accuracy of suspected stroke [30,33,34]. We believe that inclusion of NGAL, MMP-9 and the complex to the growing list of stroke biomarkers will certainly enhance the sensitivity and specificity of stroke diagnosis.

\section{Conclusion}

Our results show that NGAL, MMP-9 and their complex are elevated after hemorrhagic stroke, suggesting a potential usage of these biomarkers to detect and distinguish different types of strokes. However, rigorous studies with larger number of patients will be necessary to define their roles as stroke biomarkers.

\section{Acknowledgment}

This work was supported by AHA 13BGIA13820011, Kent State University start-up fund and Farris Family Innovation Award to WHC. WHC conceived, directed and funded the study. WHC and YCW designed experiments and analyzed data. YCW performed Western blotting and ELISA. WHC and YCW wrote the manuscript.

\section{References}

1. Go AS, Mozaffarian D, Roger VL, Benjamin EJ, Berry JD, et al. (2013) Turner, Heart disease and stroke statistics--2013 update: a report from the American Heart Association, Circulation 127: e6-e245.

2. Thrift AG, Cadilhac DA, Thayabaranathan T, Howard G, Howard VJ, et al. (2014) Global stroke statistics. Int J Stroke 9: 6-18.

3. Al-Khoury L, Lyden PD (2004) Intravenous thrombolysis. Stroke Pathophysiology, Diagnosis, and Management, Elsevier Inc., Churchill Livingstone, New York: 919-941.

4. Lo EH, Dalkara T, Moskowitz MA (2003) Mechanisms, challenges and opportunities in stroke. Nat Rev Neurosci 4: 399-415.

5. Del Zoppo GJ, Saver JL, Jauch EC, Adams HP (2009) Jr. Expansion of the Time Window for Treatment of Acute Ischemic Stroke with Intravenous Tissue Plasminogen Activator. A Science Advisory from the American Heart Association/American Stroke Association, Stroke 40: 2945-2948.

6. Kidwell CS, Latour L, Saver JL, Alger JR, Starkman S, et al. (2008) Thrombolytic toxicity: blood brain barrier disruption in human ischemic stroke, Cerebrovasc Dis 25: 338-343.

7. Kjeldsen L, Johnsen AH, Sengeløv H, Borregaard N (1993) Isolation and primary structure of NGAL, a novel protein associated with human neutrophil gelatinase. J Biol Chem 268: 10425-10432.

8. Flower DR, North AC, Sansom CE (2000) The lipocalin protein family: structural and sequence overview. Biochim Biophys Acta 1482: 9-24.

9. Elneihoum AM, Falke P, Axelsson L, Lundberg E, Lindgarde F, et al. (1996) Leukocyte activation detected by increased plasma levels of inflammatory mediators in patients with ischemic cerebrovascular diseases, Stroke 27: 1734-1738.

10. Anwaar I, Gottsater A, Ohlsson K, Mattiasson I, Lindgarde F (1998) Increasing levels of leukocyte-derived inflammatory mediators in plasma and cAMP in platelets during follow-up after acute cerebral ischemia, Cerebrovasc Dis 8: 310-317.

11. Falke P, Elneihoum AM, Ohlsson K (2000) Leukocyte activation: relation to cardiovascular mortality after cerebrovascular ischemia. Cerebrovasc Dis 10: $97-101$.
12. Wang G, Weng YC, Han X, Whaley JD, McCrae KR, et al. (2015) Lipocalin-2 released in response to cerebral ischaemia mediates reperfusion injury in mice. J Cell Mol Med .

13. Kjeldsen L, Cowland JB, Borregaard N (2000) Human neutrophil gelatinase-associated lipocalin and homologous proteins in rat and mouse, Biochim Biophys Acta 1482: 272-283.

14. Yan L, Borregaard N, Kjeldsen L, Moses MA (2001) The high molecular weight urinary matrix metalloproteinase (MMP) activity is a complex of gelatinase B/MMP-99 and neutrophil gelatinase-associated lipocalin (NGAL). Modulation of MMP-9 activity by NGAL, J Biol Chem 276: 37258-37265.

15. Fernandez CA, Yan L, Louis G, Yang J, Kutok JL, et al. (2005) The matrix metalloproteinase-9/neutrophil gelatinase-associated lipocalin complex plays a role in breast tumor growth and is present in the urine of breast cancer patients, Clin Cancer Res 11: 5390-5395.

16. Gupta K, Shukla M, Cowland JB, Malemud CJ, Haqqi TM (2007) Neutrophil gelatinase-associated lipocalin is expressed in osteoarthritis and forms a complex with matrix metalloproteinase 9. Arthritis Rheum 56: 3326-3335.

17. Brinckerhoff CE, Matrisian LM (2002) Matrix metalloproteinases: a tail of a frog that became a prince. Nat Rev Mol Cell Biol 3: 207-214.

18. Rosenberg GA (2002) Matrix metalloproteinases in neuroinflammation. Glia 39: 279-291.

19. Rosell A, Cuadrado E, Ortega-Aznar A, Hernandez-Guillamon M, Lo $\mathrm{EH}$, et al. (2008) MMP-9-positive neutrophil infiltration is associated to blood-brain barrier breakdown and basal lamina type IV collagen degradation during hemorrhagic transformation after human ischemic stroke, Stroke 39: 1121-1126.

20. Adibhatla RM, Hatcher JF (2008) Tissue plasminogen activator (tPA) and matrix metalloproteinases in the pathogenesis of stroke: therapeutic strategies. CNS Neurol Disord Drug Targets 7: 243-253.

21. McColl BW, Rothwell NJ, Allan SM (2008) Systemic inflammation alters the kinetics of cerebrovascular tight junction disruption after experimental stroke in mice, J Neurosci 28: 9451-9462.

22. Cuadrado E, Ortega L, Hernández-Guillamon M, Penalba A, FernándezCadenas I, et al. (2008) Tissue plasminogen activator (t-PA) promotes neutrophil degranulation and MMP-9 release. J Leukoc Biol 84: 207-214.

23. Gautier S, Ouk T, Petrault O, Caron J, Bordet R (2009) Neutrophils contribute to intracerebral haemorrhages after treatment with recombinant tissue plasminogen activator following cerebral ischaemia, Br J Pharmacol 156: 673-679.

24. Montaner J, Alvarez-Sabin J, Molina CA, Angles A, Abilleira S, et al. (2001) Matrix metalloproteinase expression is related to hemorrhagic transformation after cardioembolic stroke, Stroke 32: 2762-2767.

25. Abilleira S, Montaner J, Molina CA, Monasterio J, Castillo J, et al. (2003) Matrix metalloproteinase-9 concentration after spontaneous intracerebral hemorrhage. J Neurosurg 99: 65-70.

26. Kidwell CS, Chalela JA, Saver JL, Starkman S, Hill MD, et al. (2004) Comparison of MRI and CT for detection of acute intracerebral hemorrhage. JAMA 292: 1823-1830.

27. Baird AE, Dambrosia J, Janket S, Eichbaum Q, Chaves C, et al. (2001) A three-item scale for the early prediction of stroke recovery. Lancet 357 : 2095-2099.

28. Reynolds MA, Kirchick HJ, Dahlen JR, Anderberg JM, McPherson PH, et al. (2003) Early biomarkers of stroke. Clin Chem 49: 1733-1739.

29. Whiteley W, Chong WL, Sengupta A, Sandercock P (2009) Blood markers for the prognosis of ischemic stroke: a systematic review. Stroke 40: e380-389.

30. Jickling GC, Sharp FR (2011) Blood biomarkers of ischemic stroke. Neurotherapeutics 8: 349-360.

31. Foerch C, Montaner J, Furie KL, Ning MM, Lo EH (2009) Invited article: searching for oracles? Blood biomarkers in acute stroke. Neurology 73 : 393-399.

32. Lynch JR, Blessing R, White WD, Grocott HP, Newman MF, et al. (2004) Novel diagnostic test for acute stroke. Stroke 35: 57-63. 
Citation: Weng YC, Chou WH (2015) Neutrophil Gelatinase-Associated Lipocalin and Matrix Metalloproteinase-9 as Potential Biomarkers for Stroke: A Pilot Study. J Neurol Neurophysiol 6: 278. doi:10.4172/2155-9562.1000278

Page 5 of 5

33. Laskowitz DT, Kasner SE, Saver J, Remmel KS, Jauch EC (2009) B.S Group, Clinical usefulness of a biomarker-based diagnostic test for acute stroke: the Biomarker Rapid Assessment in Ischemic Injury (BRAIN) study, Stroke 40: 77-85.
34. Saenger AK, Christenson RH (2010) Stroke biomarkers: progress and challenges for diagnosis, prognosis, differentiation, and treatment. Clin Chem 56: 21-33. 